Draft version September 16, 2021

Preprint typeset using $\mathrm{IAT}_{\mathrm{E}} \mathrm{X}$ style emulateapj v. 5/2/11

\title{
A UNIVERSAL SFDM HALO MASS FOR THE ANDROMEDA AND MILKY WAY'S DSPHS?
}

\author{
V. LORA ${ }^{1}$ \\ Astronomisches Rechen-Institut, Zentrum für Astronomie der Universität Heidelberg, \\ Mönchhofstr. 12-14, 69120 Heidelberg, Germany \\ Draft version September 16, 2021
}

\begin{abstract}
Dwarf spheroidal galaxies are the most common type of galaxies, and are the most dark matter dominated objects in the Universe. Therefore, they are ideal laboratories to test any dark matter model. The Bose-Einstein condensate/scalar field dark matter model considers that the dark matter is composed by spinless-ultra-light particles which can be described by a scalar field. This model is an alternative to the $\Lambda$-cold dark matter model. In this work I study the kinematics of the dwarf spheroidal satellite galaxies of the Milky Way and Andromeda, under the scalar field/BEC dark matter paradigm in two limits: when the self interacting parameter is equal to zero, and when the self interacting parameter is $\gg 1$. I find that dwarf spheroidal galaxies with very high mass-to-light ratios (higher than 100) are in better agreement with an NFW mass density profile. On the other hand, dwarf spheroidal galaxies with relatively low mass-to-light ratios and high luminosities are better described with the SFDM model. Such results are very encouraging to further test alternative dark matter models using the dynamics of dwarf galaxies as a tool.
\end{abstract}

Subject headings: dark matter — galaxies: dwarf — galaxies: kinematics and dynamics — Local Group

\section{INTRODUCTION}

Dwarf spheroidal (dSph) galaxies are believed to be the most common type of galaxies in the Universe, and the building blocks of more massive galaxies in hierarchical formation scenarios. The large mass-to-light ratios of these dSph galaxies suggest that the dynamics of such galaxies are dominated by dark matter (DM). Therefore, the study of dSphs is crucial for a better understanding of the nature of DM. In particular, the dSph of the Local Group (LG) of galaxies satellites of the Milky Way (MW) and Andromeda galaxies, are good targets for study, since individual stars can be resolved and evolutionary histories can be derived in great detail.

Walker et al. (2009) proposed that all dSphs follow a universal mass profile, which means that all dSphs are embedded within a universal dark matter halo. They found the best-fit for the relationship between velocity dispersion and half-light radius for a maximum velocity $V_{\max }=13 \mathrm{~km} / \mathrm{s}$, and a scale radius, $r_{0}=150 \mathrm{pc}$, for a cored DM halo. For the NFW profile, the best fit is achieved for $V_{\max }=15 \mathrm{~km} / \mathrm{s}$, and a scale radius $r_{0}=$ 795 pc.

More recently, Collins et al. (2014) extended the previous work, incorporating kinematic information of 25 Andromeda dSph (Collins et al. 2013). They found a discrepancy between the DM density profile best-fit for the dSphs in the MW compared with that for Andromeda's dSphs (for both, cored and NFW cases). A good agreement between both MW dSphs and Andromeda dSphs samples is only reached, when three Andromeda outliers (And XIX, And XXI and And XXV) and three MW outliers (Hercules, CVnI and Sagittarius) are removed from the dwarf galaxy sample.

The discrepancy between the cuspy density profiles of DM halos predicted in simulations, with the cored den-

vlora@ari.uni-heidelberg.de sity profiles derived from observations of $\mathrm{dSph}$ galaxies and Low Surface Brightness galaxies (Bosch et al. 2000; Klevna et al. 2003; Blok \& Bosma et al. 2002; Lora et al. 2009; Walker \& Peñarrubia 2011; Amorisco \& Evans 2012; Jardel \& 2012) (the cusp/core problem), the overpopulation of dark substructure (Klypin et al. 1999), and the to-big-to-fail problem (Read et al. 2006; Boylan et al. 2011, 2012), have motivated alternative DM candidates to the $\Lambda \mathrm{CDM}$ model

An alternative model that lately has gained interest, is to consider that the DM is made of bosons described by a real (or complex) scalar field $\Phi$ : the Bose-Einstein condensate/scalar field DM model (BEC/SFDM) (Sin 1994; Ji \& Sin 1994; Jae-weon Lee \& In-guv Koh 1996; Peebles \& Vilenkin 1999; Matos et al. 2000; Guzmán \& Matos 2000; Matos et al. 2009; Magaña et al. 2012). The BEC/SFDM model is also known as Fuzzy DM (Hu et al. 2000) or recently as Wave DM (Schive et al. 2014).

A very interesting feature of the BEC/SFDM model, is that it naturally produces cored halos. The size of the cores of such halos (for a fixed self-interacting parameter $\Lambda$ ) depends on the mass of the BEC/SFDM boson and the mass of the SFDM halo $\left(M_{D M}\right)$.

The SFDM model has been proved to be very successful (Magaña \& Matos 2012). It is consistent with the anisotropies of the cosmic microwave background radiation (CMB) (Rodríguez-Montoya et al. 2010), and it can also achieve a better fit to highresolution rotation curves of low-surface-brightness galaxies (Robles \& Matos 2012), compared to the NFW (Navarro et al. 1996) profile.

Lately, Lora et al. (2012) and Lora \& Magaña (2014) used the internal stellar structures of dSph galaxies to establish a preferred range for the mass $m_{\phi}$ of the bosonic 
particle. They performed $N$-body simulations and explored how the dissolution time-scale of the cold stellar clump in Ursa Minor (UMi) and Sextans depends on $m_{\phi}$. They found that for a boson mass in the range of $\left(3<m_{\phi}<8\right) \times 10^{-22} \mathrm{eV}$, the BEC/SFDM model would have large enough cores to explain the stellar substructure in dSph galaxies.

Moreover, Diez-Tejedor (2014) find a preferred scale radius of $\sim 0.5-1 \mathrm{kpc}$, from the kinematics of the eight brightest dSphs satellites of the MW.

In this work I investigate the idea of a universal mass profile for the dSph population of the MW (Walker et al. 2009) and Andromeda (M31) under the SFDM model, and compare it with a cored and NFW profile (Collins et al. 2014).

The article is organized as follows: in 82 the SFDM model is described and the Schrödinger-Poisson system is briefly reviewed. In 83 , I discuss the universal NFW and cored DM profile for the dSphs in the MW and M31. In \$4. I describe our results, and finally, in section \$5 I discuss the results and give our conclusions.

\section{THE BEC/SFDM HALOS}

The DM halos can be interpreted as BEC/SFDM Newtonian gravitational configurations in equilibrium, which can be described by the so called Schrödinger-Poisson system, which is the Newtonian limit of the EinsteinKlein-Gordon equations

$$
\begin{gathered}
i \hbar \frac{\partial \psi}{\partial t}=-\frac{\hbar^{2}}{2 m_{\phi}} \nabla^{2} \psi+U m_{\phi} \psi+\frac{\lambda}{2 m_{\phi}}|\psi|^{2} \psi \\
\nabla^{2} U=4 \pi G m_{\phi}^{2} \psi \psi^{*} .
\end{gathered}
$$

The critical mass for such configurations is $M_{\text {crit }} \sim$ $0.6\left(\frac{m_{P}}{m_{\phi}}\right) \sim 10^{12} \mathrm{M}_{\odot} \quad$ (Ruffini \& Bonazzola 1969; Seidel \& Suen 1991).

In Equations 1 and 2, $m_{\phi}$ is the mass of the boson associated with the wave function $\psi$. $U$ is the gravitational potential (produced by the mass density source $\rho=m_{\phi}^{2}|\psi|^{2}$ ), and $\lambda$ is the self-interacting parameter. It has been shown that the latter term determines the compactness of the structure Guzmán \& Ureña-López 2006).

It is convenient to work with the dimensionless Schrödinger-Poisson system, given as follows (Bernal 2007)

$$
\begin{gathered}
i \frac{\partial \hat{\psi}}{\partial \hat{t}}=-\frac{1}{2} \hat{\nabla}^{2} \hat{\psi}+\hat{U} \hat{\psi}+\hat{\Lambda}|\hat{\psi}|^{2} \hat{\psi}, \\
\hat{\nabla}^{2} \hat{U}=\hat{\psi} \hat{\psi}^{*}
\end{gathered}
$$

where

$$
\Lambda=\frac{\lambda}{4 \pi m_{\phi}^{2} G}
$$

The solutions to the Schrödinger-Poisson system relevant for this work are those with spherical equilibrium (Chi-Wai Lai 2004; Bernal 2007). Hence, it is convenient to work with the Schrödinger-Poisson system in spherical coordinates

$$
\begin{gathered}
i \frac{\partial \hat{\psi}}{\partial \hat{t}}=-\frac{1}{2 \hat{r}} \frac{\partial^{2}}{\partial \hat{r}}(\hat{r} \hat{\psi})+\hat{U} \hat{\psi}+\hat{\Lambda}|\hat{\psi}|^{2} \hat{\psi}, \\
\frac{\partial^{2}}{\partial \hat{r}}(\hat{r} \hat{U})=\hat{r} \hat{\psi} \hat{\psi}^{*} .
\end{gathered}
$$

The solutions of the Schrödinger-Poisson system are obtained assuming an harmonic behavior of the scalar field, such that

$$
\hat{\psi}(r, t)=e^{-(i \hat{\gamma} \hat{t})} \hat{\phi}(\hat{r}),
$$

where $\hat{\gamma}$ is an adimensional frequency.

Substituting $\hat{\psi}$ in Equations 6 and 7 , the SchrödingerPoisson system now reads as

$$
\begin{gathered}
\frac{d^{2}}{d \hat{r}^{2}}(\hat{r} \hat{\phi})=2 \hat{r}(\hat{U}-\hat{\gamma})+2 \hat{r} \Lambda \hat{\phi}^{3} \\
\frac{d^{2}}{d \hat{r}^{2}}(\hat{r} \hat{U})=\hat{r} \hat{\phi}^{2}
\end{gathered}
$$

The SFDM halos are constructed by obtaining ground state (stable) solutions of the Schrödinger-Poisson system (9) 10) Guzmán \& Ureña-López 2004; Bernal 2007; Lora et al. 2012; Lora \& Magaña 2014). To guarantee regular solutions, the boundary conditions must satisfy that at $r=0 \partial_{r} U=0, \partial_{r} \phi=0$, and that $\phi(0)=\phi_{c}=$, where $\phi_{c}$ is an arbitrary value.

The mass of this BEC/SFDM halo can be estimated as

$$
M=4 \pi \int_{0}^{\infty} \phi^{2} r^{2} d r,
$$

and must be finite number. The radius of this configuration is defined as $r_{95}$, which is the radius containing $95 \%$ of the mass. Note that both properties, the mass and the radius of the $\mathrm{SF}$ halo depend on the boson mass, and the self-interacting term. Three parameters $\phi_{c}, m_{\phi}$ and $\Lambda$, define a model completely.

\subsection{The $\Lambda \gg 1$ case}

When the self interaction between the bosons in a Bose-Einstein condensate is taken into account, the Schrödinger equation can be interpreted as the meanfield approximation at zero temperature of the GrossPitaevskii equation (see Equation 11). The limit where the number of particles in the BEC is very large, and thus the self interacting term dominates, is called the ThomasFermi limit (TFL) (Pitaevskii 1961; Dalfovo et al. 1999).

As the number of particles in the BEC becomes infinite, the TFL approximation becomes exact (Barcelo et al. 2005), giving as a result the classical limit of the theory. Then, the equations describing the static $\mathrm{BEC}$ in a gravitational field with potential $U$ take the following form (Böhemer \& Harko 2007):

$$
\begin{aligned}
\nabla P\left(\frac{\rho}{m_{\phi}}\right) & =-\rho \nabla\left(\frac{U}{m}\right), \\
\nabla^{2} U & =4 \pi G \rho .
\end{aligned}
$$

With appropriate boundary conditions, one can integrate the latter equations, assuming an equation of 


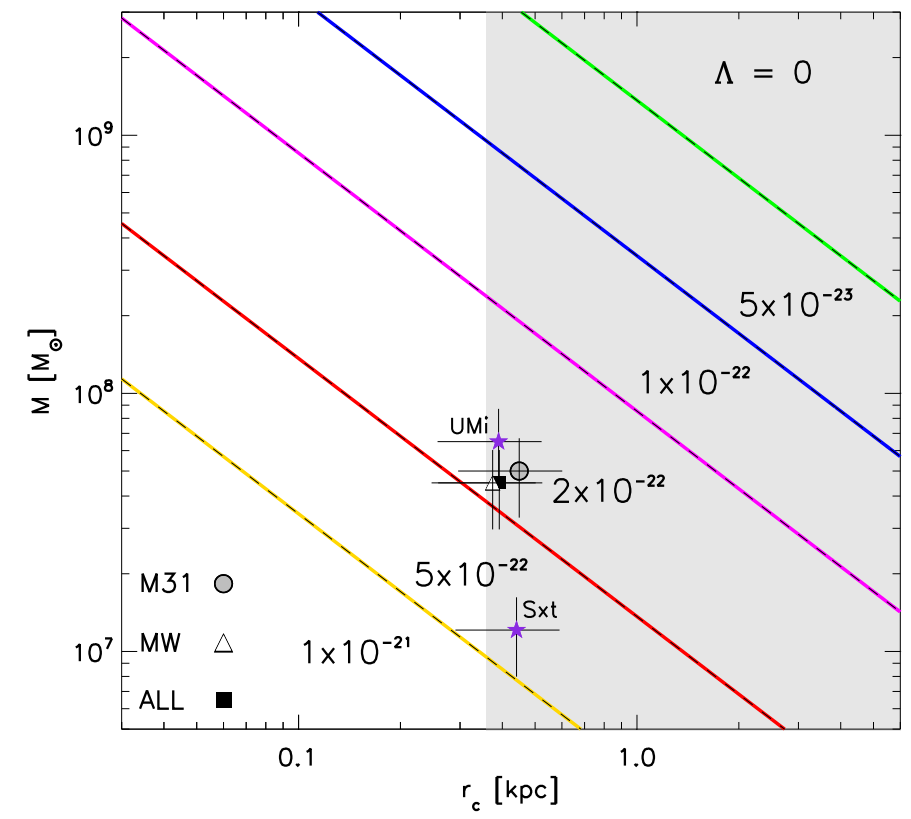

Fig. 1. - Mass of the SFDM halo, as a function of the SFDM core radius for the $\Lambda=0$ case. Each diagonal line corresponds to a different value of the SFDM boson $m_{\phi}: 5 \times 10^{-23}$ (green), $10^{-22}$ (blue), $2 \times 10^{-22}$ (pink), $5 \times 10^{-22}$ (red), and $10^{-21}$ (yellow) eV. The shaded region shows the permitted core radius $r_{c}$, such that the stellar clumps in Ursa Minor and Sextans are not destroyed (Lora et al. 2012; Lora \& Magaña 2014).

state of the form $P=P(\rho)$. Assuming the nonlinearity in the Gross-Pitaevskii equation of the form $g(\rho)=\alpha \rho^{\Gamma}$ (where $\alpha$ and $\Gamma$ are constants greater than zero) (Böhemer \& Harko 2007), then the BEC equation of state is given by

$$
P=P(\rho)=\alpha(\Gamma-1) \rho^{\Gamma} .
$$

If $\Gamma$ is represented by $\Gamma=1+\frac{1}{n}$ (where $n$ is the polytropic index), then the equation of state of the static gravitationally bounded BEC is described by the LaneEmden equation

$$
\frac{1}{\xi^{2}} \frac{d}{d \xi}\left(\xi^{2} \frac{d \theta}{d \xi}\right)+\theta^{n}=0 .
$$

In particular, for a static BEC in the TFL with a politropic index $n=1$, the Gross-Pitaevskii equation reduces to a Lane-Emden equation of the form

$$
\frac{d^{2} \theta}{d \xi^{2}}+\frac{2}{\xi} \frac{d \theta}{d \xi}+\theta=0
$$

with an analytical solution

$$
\theta(\xi)=\frac{\sin \xi}{\xi} .
$$

Using the solution given by Böhemer \& Harko (2007) for the density profile, one has

$$
\rho(r)= \begin{cases}\rho_{0} \frac{\sin \left(\pi r / R_{\max }\right)}{\pi r / R_{\max }} & \text { if } r \leq R_{\max } \\ 0 & \text { if } r>R_{\max },\end{cases}
$$

where $R_{\max }$ can be interpreted as the size of the core radius of the SFDM halo.
Finally, from Equation 11] one can obtain the mass within a radius $r$ in the TFL

$M(r)=\frac{4}{\pi} \frac{\rho_{0} R_{\max }^{3}}{r}\left[\sin \left(\frac{\pi r}{R_{\max }}\right)-\frac{\pi r}{R_{\max }} \cos \left(\frac{\pi r}{R_{\max }}\right)\right]$.

Thus, the quantities that describe a system completely in the $\Lambda \gg 1$ limit (TFL) are the central mass density $\rho(c)$ and the size of the SFDM halo $R_{\max }$.

As Diez-Tejedor (2014) point out, the size of the DM halos in the TFL can be expressed as

$$
R_{\max }=48.93\left(\frac{\lambda^{1 / 4}}{m_{\phi}}\right)^{2}
$$

showing the dependency of $R_{\max }$ with $\left(\frac{m_{\phi}}{\lambda^{1 / 4}}\right)$.

\section{NFW AND CORED PROFILES FOR THE MILKY WAY AND ANDROMEDA DSPHS}

The measurements of central velocity dispersions of dSph galaxies very well constrain the dynamical mass within the deprojected half-light radii $\left(M_{1 / 2}\right)$ (Walker et al. 2009; Wolf et al. 2010), then one can compare such dSph mass $\left(M_{1 / 2}\right.$ Vs $\left.r_{1 / 2}\right)$, with a certain integrated DM mass profile, given a total DM halo mass $M$.

Walker et al. (2009) applied the Jeans equation to the velocity dispersion profiles of eight of the brightest $\mathrm{dSph}$ galaxies of the MW and consider the hypothesis that all dSphs follow a universal mass profile, i.e. that all dSphs are embedded within a universal dark matter halo.

Using the density Equation,

$$
\rho(r)=\frac{\rho_{0}}{\left(r / r_{0}\right)^{\alpha}\left(1+r / r_{0}\right)^{\beta-\alpha}},
$$


Walker et al. (2009) fit a cored $(\alpha=0, \beta=3)$ and an NFW (Navarro et al. 1996) $(\alpha=1, \beta=3)$ DM profile. For the cored DM halo, they found the best-fit for the relationship between velocity dispersion and the halflight radius, for a maximum velocity $V_{\max }=13 \mathrm{~km} / \mathrm{s}$, and a scale radius, $R_{S}=150 \mathrm{pc}$. For the NFW profile, the best fit is achieved for $V_{\max }=15 \mathrm{~km} / \mathrm{s}$, and a scale radius $R_{S}=795 \mathrm{pc}$.

Collins et al. (2014) extended the idea of a universal mass profile, now including the M31 objects into the analysis (Collins et al. 2013).

Collins et al. (2014) also use the NFW and core mass density profiles. They obtain the best fit for the NFW (cored) profile, to the whole population of dSphs, of $V_{\max }=14.7 \pm 0.5 \mathrm{~km} / \mathrm{s}\left(V_{\max }=14.0 \pm 0.4 \mathrm{~km} / \mathrm{s}\right)$ and $\mathrm{a}$ scale radius of $R_{S}=876 \pm 284 \mathrm{pc}\left(R_{S}=242 \pm 124 \mathrm{pc}\right)$. They concluded that neither value is a good fit for many of the LG dSphs. They found that if they do not take into account five outliers, three in M31 (And XIX, And XXI, and And XXV), and two in the MW (Hercules and $\mathrm{CVn} \mathrm{I}$ ), the fit is significantly better for the NFW (core) mass profile, $V_{\max }=16.2_{-1.7}^{+2.6} \mathrm{~km} / \mathrm{s}\left(V_{\max }=\right.$ $\left.15.6_{-1.3}^{+1.5} \mathrm{~km} / \mathrm{s}\right)$ and $R_{S}=664_{-232}^{+412} \mathrm{pc}\left(R_{S}=225_{-55}^{+70} \mathrm{pc}\right)$.

\section{A UNIVERSAL SFDM HALO?}

\subsection{The $\Lambda=0$ case}

One can consider the self-interacting term $\Lambda$ to be zero in Equation 1 (i. e. the self-interaction is negligible), such a case is also known as the fuzzy DM model (Hu et al. 2000).

In Figure 1, the mass of the SFDM halo is plotted as a function of the core radius $\left(r_{c}\right)$ for $\Lambda=0$. The core radius is defined as the radius at which the initial density has dropped by a factor of two.

When analyzing the behavior of the different models with fixed $M_{D M}$ and $m_{\phi}$, I found out that there is a correlation between the mass of the SFDM halo and the core radius, for a given $m_{\phi}$. The mass of the SFDM halo is inversely proportional to the DM core radius independently of the value of $m_{\phi}$ (i. e. $M_{D M} \propto r_{c}^{-1}$ ), but the intercept varies depending on the value of $m_{\phi}$. Then, I found a fit over different values of $m_{\phi}$ and their corresponding intercepts $(\alpha)$, and found the relation between the intercept and the value of $m_{\phi}$ of $\alpha \propto m_{\phi}^{8.7}$.

The complete relation between the mass of the dark matter halo and the dark matter halo's core radius for a given $m_{\phi}$, is given by

$$
M_{D M}=10^{\alpha} r_{c}^{-1}
$$

where $\alpha$ depends on the mass of the boson as

$$
m_{\phi}=10^{-13.9} \alpha^{-8.7} \text {. }
$$

For a given $M_{D M}$ and $m_{\phi}$, there is a slope $\alpha$, for which a unique $r_{c}$ will be defined. With the latter relations, one can build a set of continuous models for a given $M_{D M}$ and $m_{\phi}$. The continuous models are shown as diagonal colored lines in Figure 11. Each color represent a mass of the boson $m_{\phi}$ (green $\rightarrow 5 \times 10^{-23} \mathrm{eV}$, blue $\rightarrow 1 \times$ $10^{-22} \mathrm{eV}$, pink $\rightarrow 2 \times 10^{-22} \mathrm{eV}$, red $\rightarrow 5 \times 10^{-22} \mathrm{eV}$, yellow $\rightarrow 1 \times 10^{-21} \mathrm{eV}$ ).
It is clear to see that for a given halo mass, lower values of $m_{\phi}$ 's have larger core-radius, and higher values of $m_{\phi}$ have smaller core-radius. For example, for a fixed SFDM halo mass of $10^{8} M_{\odot}$, if $m_{\phi}=10^{-22} \mathrm{eV}$ the corresponding core radius is $\sim 3.5 \mathrm{kpc}$. But if $m_{\phi}=10^{-21} \mathrm{eV}$ the corresponding core radius is $\sim 30 \mathrm{pc}$.

I consider three different groups of dSph data; the dSphs in the MW (19 galaxies), the dSph in the M31 (22 galaxies), and ALL the galaxies (41 galaxies). In order to compare with Collins et al. (2014) results, I do not take into account five dSphs (Sagittarius, Hercules, CvnI, AndXIX, AndXXI and And XXV).

In order to see how well these three groups of dSph galaxies can be fit with a single SFDM profile (for a SFDM halo mass $M$ and a SFDM boson mass $m_{\phi}$ ), I use the following maximum likelihood fitting routine

$$
\begin{aligned}
& L_{S F D M}\left(\left\{r_{h, i}, V_{c, i}, \delta_{V_{c}, i}\right\} \mid m_{\phi}, M\right)= \\
& \quad \prod_{i=0}^{N} \frac{1}{\sqrt{2 \pi} \delta_{V_{c}, i}} \times \exp \left[-\frac{\left(V_{c, S F D M}-V_{c, i}\right)^{2}}{2 \delta_{V_{c}, i}^{2}}\right],
\end{aligned}
$$

where $V_{c, S F D M}$ is the circular velocity predicted by the SFDM model, $r_{h, i}$ is the half-light radius of the $i-\mathrm{dSph}$, $V_{c, i}$ is the measured circular velocity at the half-light radius, and $\delta_{V_{c}, i}$ is its uncertainty (the observational data are taken from McConnachie 2012, Collins et al. 2014 and Martin et al. 2015).

Equation 24 is a measure of how well a model with specific parameters $m_{\phi}$ and $M$ represents a given group of galaxies. In order to find the best fit for the three samples I use the Evolution Strategy with Covariance Matrix Adaptation (CMA-ES) optimization. For details on the CMA-ES routine, see Appendix A.

The best fit for the 19 MW sample is $M=5.12 \times$ $10^{7} M_{\odot}$ and a $m_{\phi}=3.8 \times 10^{-22} \mathrm{eV}$. For the $22 \mathrm{M} 31$ sample the best fit corresponds to $M=4.44 \times 10^{7} M_{\odot}$ and a $m_{\phi}=4.53 \times 10^{-22} \mathrm{eV}$. For the whole sample (41) the best fit corresponds to $M=4.8 \times 10^{7} M_{\odot}$ and $m_{\phi}=$ $4.17 \times 10^{-22} \mathrm{eV}$ (see Table 1). The MW fit gives as a result a slightly more massive DM halo, and a smaller mass of the boson, compared with the M31 and the ALL data sets. As a consequence the core radius for the MW sample, is the largest $\left(r_{c} \sim 0.45 \mathrm{kpc}\right)$. Such a size of the DM core radius is large enough to explain the longevity of the old-cold stellar clump in UMi (Lora et al. 2012), and the two stellar substructures in Sextans (Lora \& Magaña 2014).

In the upper panels of Figure 2, I show the half-light radius circular velocity $\left(V_{c, 1 / 2}=\sqrt{\frac{G M_{1 / 2}}{r_{1 / 2}}}\right)$ as a function of the half-light radius of all M31 and MW dSphs in the sample, along with the best fit to ALL sample for an NFW density mass profile (see dashed dark-blue line) and a cored density mass profile (see dashed purple lines) (Collins et al. 2014), and the SFDM profile (see light-blue dash line). The shaded regions in Figure 2 indicate the $1 \sigma$ error. The computed maximum circular velocity for the SFDM model for the ALL sample is $V_{\max } \approx 16.63 \mathrm{~km} / \mathrm{s}$. This value of the maximum circular velocity is in a very good agreement with both computed values of the NFW profile $\left(V_{\max }=16.2_{-1.7}^{+2.6} \mathrm{~km} / \mathrm{s}\right)$, and the core profile $\left(V_{\max }=15.6_{-1.3}^{+1.5} \mathrm{~km} / \mathrm{s}\right)$. 
TABLE 1

Parameters of the best fit after a CMA optimization to the SFDM profile for three groups: the MW DSph(19), the M31 DSph(22), AND ALL the SAmple(41).

\begin{tabular}{lccccccc}
\hline Group & $\begin{array}{c}\text { Number } \\
\text { of galaxies }\end{array}$ & $\begin{array}{c}\mathrm{M} \\
{\left[10^{7} \mathrm{M} \odot\right]}\end{array}$ & $\begin{array}{c}\mathrm{m}_{\phi} \\
{\left[10^{-22} \mathrm{eV}\right]}\end{array}$ & $\begin{array}{c}\epsilon \\
10^{-5}\end{array}$ & $\begin{array}{c}r_{95} \\
{[\mathrm{kpc}]}\end{array}$ & $\begin{array}{c}\mathrm{V}_{c} \\
{[\mathrm{~km} / \mathrm{s}]}\end{array}$ & $\begin{array}{c}r_{\text {core }} \\
{[\mathrm{kpc}]}\end{array}$ \\
\hline & & & & & & & \\
M31 & 22 & 5.12 & 3.80 & 7.07 & 0.90 & 16.15 & 0.46 \\
MW & 19 & 4.44 & 4.54 & 7.31 & 0.73 & 16.71 & 0.37 \\
ALL & 41 & 4.80 & 4.17 & 7.28 & 0.80 & 16.63 & 0.41 \\
& & & & & & & \\
\hline
\end{tabular}
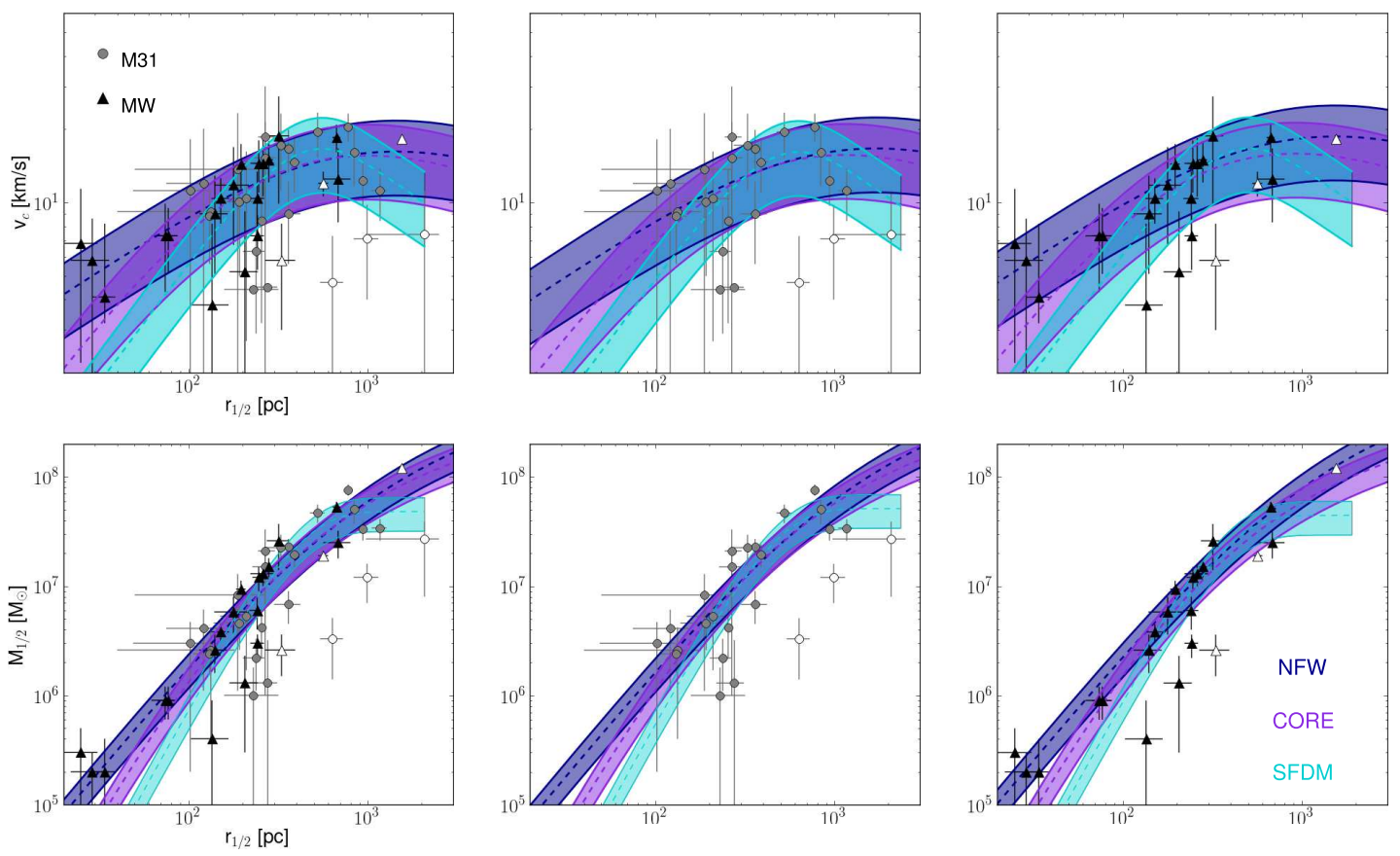

FIG. 2.- Best fit to the NFW (dark blue), and the core (purple) DM density profile from Collins et al. (2014), leaving out the MW dSphs' outliers (Hercules, CVnI and Sagittarius, see white triangles), and three M31 outliers M31 (AndXIX, AndXXI, AndXXV, white circles). The SFDM profile studied in this work, is shown in light-blue. The shaded regions correspond to a $1 \sigma$ deviation, from each DM model fit. The upper panels show the circular velocity as a function of the half-light radius, for ALL, M31, and MW samples, respectively. The lower panels show the DM halo mass, as a function of the half-light radius, for ALL, M31, and MW samples, respectively. The dSph galaxies used in this work are over plotted with their corresponding uncertainties. The gray circles correspond to the M31 dwarfs, and the black triangles correspond to the MW dSphs.

The dSph galaxies with low $V_{c} \mathrm{~s}$ and small half-light radii can be well reproduced with the cored and the NFW density profile, but cannot be well reproduced with the SFDM profile. On the other hand, the dSphs with $V_{c} \mathrm{~s}$ between $6-10 \mathrm{~km} / \mathrm{s}$, and high values of the half-light radii $(\sim 1000 \mathrm{pc})$ can be reproduced with the SFDM model, but cannot be well reproduced with either the NFW profile, or with the core profile.

In the lower panels of Figure 2, I show the same fits (core, NFW and SFDM) for the mass of the DM halo, as a function of the half-light radius. It is very clear from this Figure, that the NFW fits the ALL data better at small-mass-small-half-light-radius, whereas the SFDM fits better the ALL data at high-mass-high-half-light- radius, where both core and NFW profiles fail.

In order to look in more detail at the properties of the dSphs that are in better agreement with the SFDM profile, I plot the mass inside the half-light radius as a function of the luminosity at the same radius (see left panel of Figure 3). The diagonal dashed lines in Figure 3 correspond to four fixed mass-to-light ratios $(M / L=1000$, 100, 15 and 3). The circles represent the M31 dSphs, and the triangles represent the MW dSphs.

For each dSph I computed the ratio $\zeta$ between the theoretical mass values from the best DM fits of the ALL data set, and the observed mass values (Collins et al. 2014), $\zeta=M_{D M} / M_{d S p h}$. I then define that a dSph is well reproduced by a certain DM model if $\zeta$ is at most 2. The 
latter means that the dSph mass obtained from the best DM model differs from the observed value by a maximum of a factor 2. A dSph galaxy is in agreement with an specific DM model when $\zeta$ is minimized.

In the left panel of Figure 3 , the color code is as follows: the light-blue symbols (both M31 circles and MW triangles) represent the dSphs which are in good agreement with the SFDM profile. The purple symbols show the dSphs which are better reproduced with the cored DM profile, and the dark-blue symbols represent the dSphs which are in better agreement with the NFW profile. On the other hand, the yellow symbols correspond to the $\mathrm{dSph}$ that are well reproduced with the three DM models, whereas the red symbols show the dwarf galaxies that cannot be reproduced with any DM model studied in this work (i. e. $\zeta>2$ ).

One can immediately see from Figure 3 that the dSphs which are in a better agreement with an NFW DM profile are very dark matter dominated, with mass-to-light ratios between $1600 \gtrsim M / L \gtrsim 100$. There are only two exceptions LeoI and LeoII, which mass-to-light ratios of 7.1 and 12.9 , respectively.

The galaxies which are better reproduced with an NFW profile are also the less luminous of the sample with $L \lesssim 10^{4} \mathrm{~L}_{\odot}$. The only outlier is UMaI, which is in a better agreement with the SFDM model. On the other hand, it seems that these galaxies have a mass at the half-light ratio limit of $\sim 10^{7} \mathrm{M}_{\odot}$.

The upper-right gray shaded region in Figure 3 contains a set of five M31 dSphs, and two MW dSphs which are in good agreement with the SFDM profile (light-blue symbols). If one also takes into account the yellow symbols, which are dSphs well reproduced with either DM model, then four more can be added, resulting in a set of 11 galaxies which are very well reproduced with the SFDM model. These galaxies share the property of being less DM dominated than those which are better reproduced with an NFW DM profile. The mass-luminosity ratios of these galaxies range from $\sim 100$ to 5 . On the other hand, these dSphs are the most luminous galaxies of the sample, with a luminosity ranging from $\sim 10^{5}$ to $5 \times 10^{6} \mathrm{~L} \odot$. It is remarkable that there are no dSph in this region which are more in agreement with an NFW profile.

\subsection{The $\Lambda>>1$ case}

In this subsection, the kinematics of the dSphs in the MW and M31, for large values of the self-interacting parameter $\Lambda$, are analyzed. In the $\Lambda \gg 1$ limit, as mentioned in section 4.2, the SFDM density profile is described by Equation 18, In order to build the DM halos the value of the central DM density $\rho_{0}$ and the size of the SFDM halo $R_{\max }$ must be fixed.

To study how well the circular velocity, and mass profile of the three groups of dSphs (M31, MW and ALL) are fit with a single SFDM halo in $\Lambda>>1$ regime the maximum likelihood fitting routine is modified, so that $\rho_{0}$ and $R_{\max }$ are the free parameters. Then similarly to Equation 24 one has

$$
\begin{aligned}
& L_{T F}\left(\left\{r_{h, i}, V_{c, i}, \delta_{V_{c}, i}\right\} \mid \rho_{0}, R_{\max }\right)= \\
& \quad \prod_{i=0}^{N} \frac{1}{\sqrt{2 \pi} \delta_{V_{c}, i}} \times \exp \left[-\frac{\left(V_{c, T F}-V_{c, i}\right)^{2}}{2 \delta_{V_{c}, i}^{2}}\right],
\end{aligned}
$$

where $V_{c, T F}$ is the circular velocity in the TFL.

Using the CMA-ES optimization routine described in Appendix A. I find that for the M31 dwarfs, the best fit is achieved for a value of $m_{\phi}^{4} / \lambda=3180$, which corresponds to a $\rho_{0} \sim 8 \times 10^{7} \mathrm{M}_{\odot} \mathrm{kpc}^{-3}$, a $R_{\max }=0.87 \mathrm{kpc}$, and at circular velocity value of $18.24 \mathrm{~km} / \mathrm{s}$ (see Table 21). The latter value is approximately $13 \%$ higher than the one obtained for the $\Lambda=0$ case $(16.1 \mathrm{~km} / \mathrm{s})$. The core radius (halo DM mass $M$ ) in the TFL for M31 dwarfs, is $0.52 \mathrm{kpc}\left(6 \times 10^{7} \mathrm{M}_{\odot}\right)$, very similar to the $0.45 \mathrm{kpc}$ $\left(5 \times 10^{7} \mathrm{M}_{\odot}\right)$ find for the $\Lambda=0$ case. The results are summarized in Table 2 .

For the MW dwarfs the central density is the highest of the three groups, $1.8 \times 10^{8} \mathrm{M}_{\odot} \mathrm{kpc}^{-3}$, whereas the maximum DM radius for the MW dSphs is the smallest of the three groups, $R_{\max }=0.59 \mathrm{kpc}$ (which corresponds to a core radius of $0.36 \mathrm{kpc}$ ). Such a core radius is very similar to the one obtained for the $\Lambda=0$ case, and thus, it is large enough to explain the stellar substructures found within the UMi and the Sextans dSphs. The maximum circular velocity is $18.6 \mathrm{~km} / \mathrm{s}$, again approximately $11 \%$ higher than that found in the $\Lambda=0$ case $(16.7 \mathrm{~km} / \mathrm{s})$. For the MW dwarfs a value of $m_{\phi}^{4} / \lambda=6788$ is obtained (i. e. $m_{\phi} / \lambda^{1 / 4} \approx 9$ ).

The upper panels of Figure 4 show the circular velocity as a function of the half-light radius, and DM halo mass as a function of the half-light radius (lower panels) for the SFDM model for $\Lambda=0$ and $\Lambda>>$. The pink lines show the best fit for the SFDM model in the TFL $(\Lambda>>1)$ for each of the dSph data sets: ALL (left panels), M31 (central panels) and MW (right panels), respectively. The shaded regions show the $1 \sigma$ deviation. The results obtained for the $\Lambda=0$ case (light-blue), are also plotted in order to facilitate comparison between both SFDM profiles.

In the right panel of Figure 3 I show the seven dSphs which are in better agreement with a SFDM model in the TFL (such galaxies are plotted with pink symbols) also including the previous studied DM profiles. The galaxies that are better reproduced with the SFDM in the TFL are also mostly contained in the upper-right shaded gray region. This means that the SFDM model in the TFL better fit galaxies with low mass-to-light ratios.

\subsection{The universal SFDM profile compared}

In Lora et al. (2012) we performed $N$-body simulations of the UMi dSph, which contains a cold-old stellar substructure (Kleyna et al. 2003). We explored how the dissolution time-scale of such stellar substructure depends on the mass of the boson $m_{\phi}$. The boson mass range obtained from UMi's dynamics is $0.3 \times 10^{-22}<m_{\phi}<$ $10^{-22} \mathrm{eV}$.

Similarly, in Lora \& Magaña (2014) we investigated the dSph Sextans, which has two different old-cold stellar substructures (Battaglia et al. 2011; Walker et al. 2006). For Sextans we require a mass for the SFDM boson of 

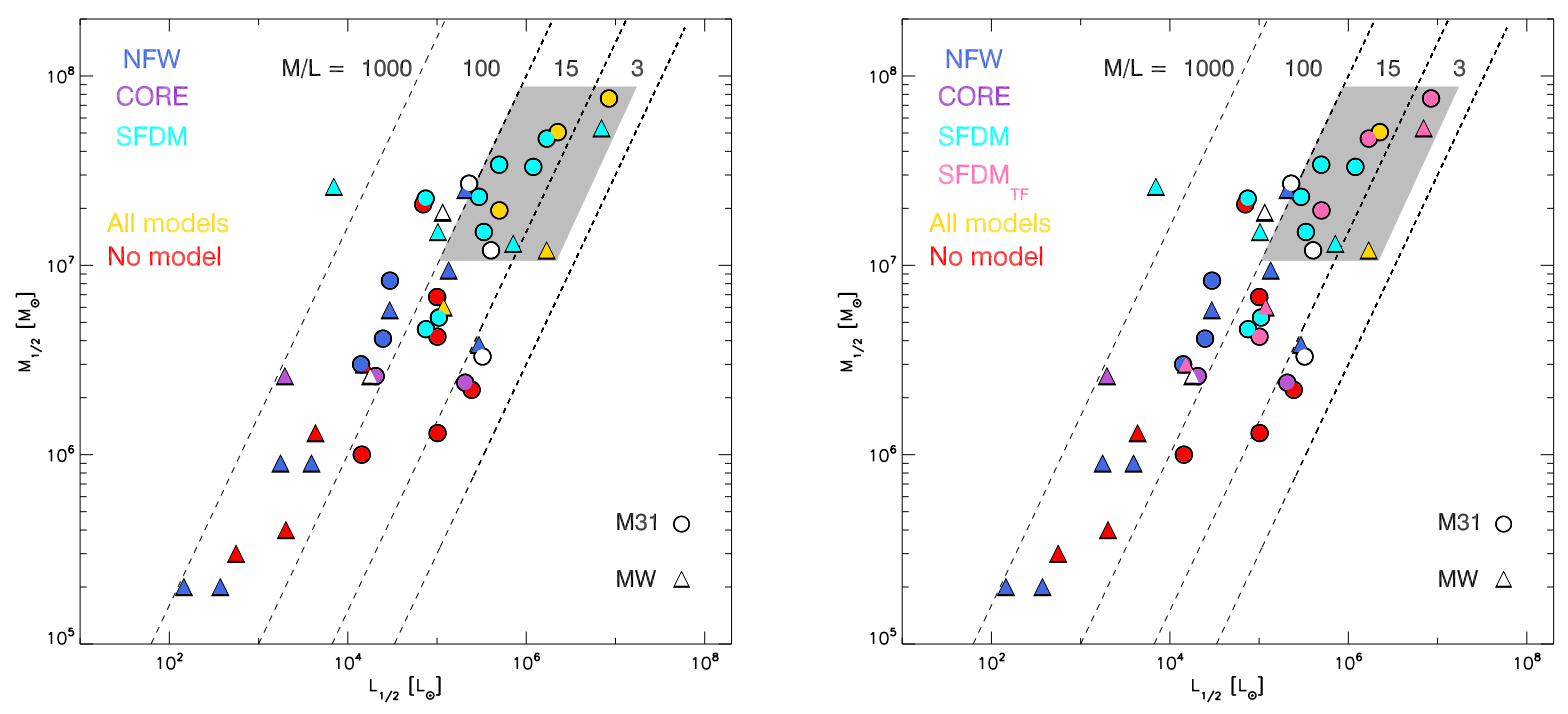

FIG. 3.- Mass at the half-light radius as a function of the luminosity at the half-light radius. The M31 dSphs are shown with circles and the MW dSphs are shown with triangles. The dark-blue(purple) symbols show the dSphs that are in better agreement with an NFW (cored) DM profile. The light-blue symbols show the dSphs which are in better agreement with the SFDM profile. The yellow symbols show the dwarfs that are well reproduced with the three DM models, and the red symbols show the dwarfs that are not well reproduced with any DM model. The white symbols represent the galaxies that were not taken into account (Hercules, CVnI, AndXIX, AndXXI and AndXXV). The diagonal dashed lines show the mass-to-light ratios $M / L=1000,100,15$, and 3 . In the right panel, the dSph that are in a better agreement with the SFDM in the TFL i. e. $\Lambda>>1$, are also plotted (see pink symbols).

TABLE 2

Parameters of the best fit in the TFL $(\Lambda>>0)$ For three Groups: the MW DSph(19), the M31 DSph(22), AND ALL the SAMPLE(41).

\begin{tabular}{|c|c|c|c|c|c|c|c|c|}
\hline Group & $\begin{array}{c}\text { Number } \\
\text { of galaxies }\end{array}$ & $\begin{array}{c}\rho_{0} \\
{\left[10^{7} \mathrm{M}_{\odot} \mathrm{kpc}^{3}\right]}\end{array}$ & $\begin{array}{r}R_{\max } \\
{[\mathrm{kpc}]}\end{array}$ & $\begin{array}{c}\mathrm{M}_{\max } \\
{\left[10^{7} \mathrm{M}_{\odot}\right]}\end{array}$ & $\begin{array}{c}\frac{m_{\phi}^{4}}{\lambda} \\
{\left[\mathrm{eV}^{4}\right]}\end{array}$ & $\begin{array}{l}\frac{m_{\phi}}{\lambda^{1 / 4}} \\
{[\mathrm{eV}]} \\
\end{array}$ & $\begin{array}{c}\mathrm{V}_{c} \\
{[\mathrm{~km} / \mathrm{s}]}\end{array}$ & $\begin{array}{l}r_{\text {core }} \\
{[\mathrm{kpc}]}\end{array}$ \\
\hline M31 & 22 & 8.06 & 0.86 & 6.716 & 3180.96 & 7.51 & 18.24 & 0.52 \\
\hline MW & 19 & 17.96 & 0.59 & 4.80 & 6788.80 & 9.08 & 18.64 & 0.36 \\
\hline ALL & 41 & 9.74 & 0.85 & 7.60 & 3325.20 & 7.59 & 19.61 & 0.51 \\
\hline
\end{tabular}

$0.12 \times 10^{-22}<m_{\phi}<8 \times 10^{-22} \mathrm{eV}$, in order to guarantee the survival of both stellar substructures.

In Figure 11 the corresponding mass of the boson is plotted (purple blue stars) for UMi and for Sextans. The error-bars denote the $1 \sigma$ deviation. The best fit of the MW(triangle), M31(circle), and ALL the sample (square) are also plotted.

For the Sextans dSph, all the values for the mass $M$ and the core radius $r_{c}$ in the light-gray area in Figure [1] are permitted. In particular, those corresponding to the three best SFDM fits (MW, M31 and ALL ). The latter means that the dynamics of the Sextans dwarf, is in agreement with a unique SFDM mass profile. The UMi dSph is located above the MW, and ALL fits in Figure 1, being the most MW dSph restrictive case. However, it is still in agreement with the MW (circle) case at $1 \sigma$ confidence.

My findings in this work in the TFL can be directly compared with the recent results of Diez-Tejedor (2014). They constrained the parameters of the SFDM selfinteracting parameter with the kinematics of the eight brightest $\mathrm{dSph}$ of the MW. They reported a preferred value of $R_{\max } \sim 1 \mathrm{kpc}$, which corresponds to $m_{\phi} / \lambda^{1 / 4} \sim$
$7 \mathrm{eV}$. In this work I obtain for the MW sample a value of $R_{\max } \sim 0.6 \mathrm{kpc}$, which corresponds to $m_{\phi} / \lambda^{1 / 4} \sim 9 \mathrm{eV}$. It has to be noted that Diez-Tejedor (2014) analyzed the eight brightest dSph in the MW; in this work the MW sample was comprised of 19 dwarf galaxies. If the MW sample is restricted to the eight brightest dSph, I obtain a value $R_{\max }=0.57 \mathrm{kpc}$, which corresponds to $m_{\phi} / \lambda^{1 / 4}=9.24 \mathrm{eV}$. This result is still in agreement with the results obtained from the $19 \mathrm{MW}$ dwarf sample, but $m_{\phi} / \lambda^{1 / 4}$ (and $R_{\max }$ ) is somewhat higher (lower) that the $m_{\phi} / \lambda^{1 / 4} \sim 7 \mathrm{eV}\left(R_{\max } \sim 1 \mathrm{kpc}\right)$ values reported by Diez-Tejedor (2014).

It has to be noted that Diez-Tejedor (2014) analyze the velocity dispersion of the eight brightest dSph galaxies in the MW, arguing that the stellar component of each of the eight galaxies are in dynamical equilibrium, and that the stellar distribution traces the DM distribution. They fit three free parameters in their models: $R_{\max }$, $M_{\max }$ and the orbital anisotropy of the stellar component of each galaxy, which is a difference in the approach of this work. It is encouraging that, even with these differences, when the uncer- 

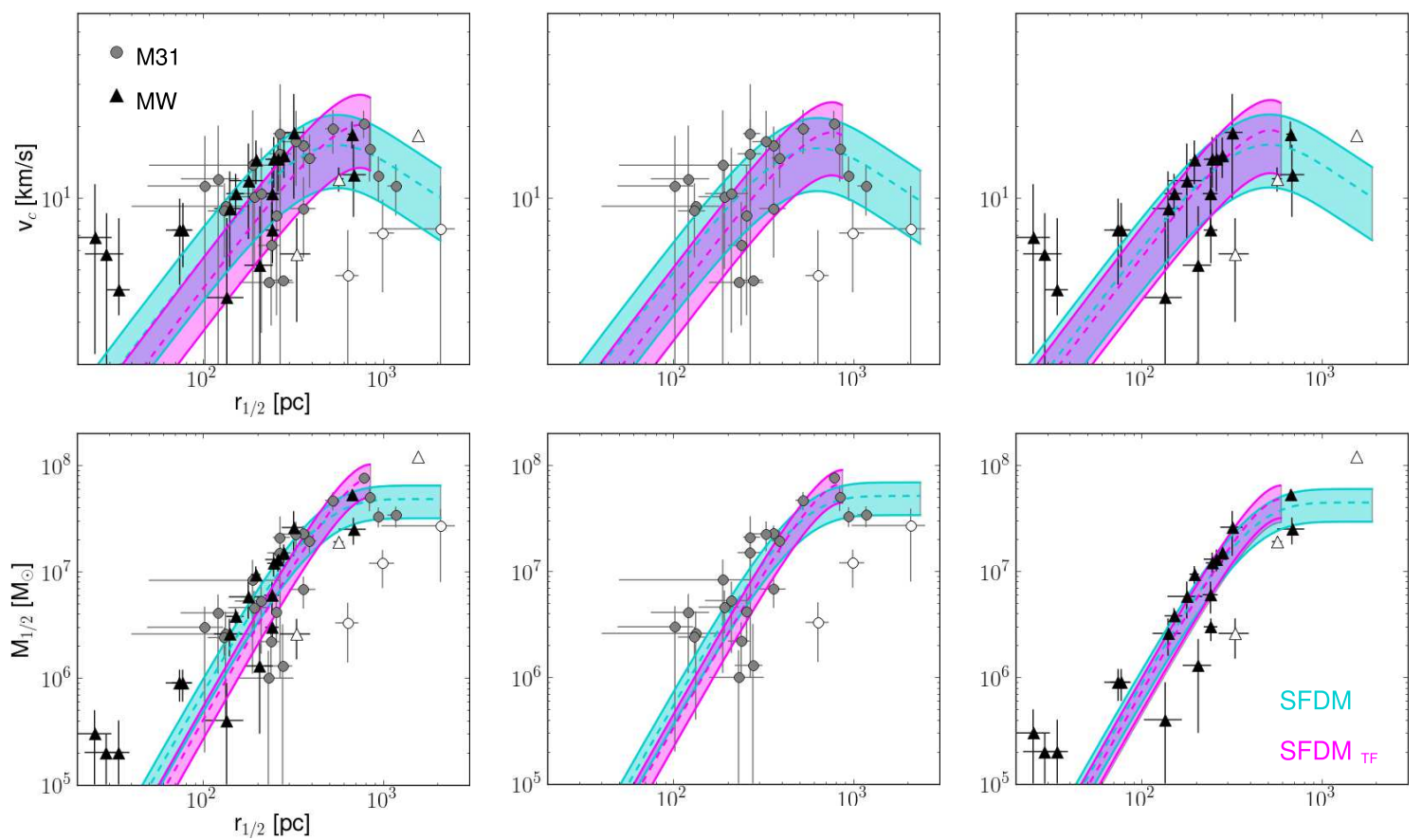

FIG. 4.- Same as Figure 2 but for the best fits of the SFDM model, and the SFDM in the TFL.

tainty averaged over the eight $\mathrm{dSphs}$ is taken into account in $R \max$ (and thus $m_{\phi} / \lambda^{1 / 4}$ ) one obtains $R_{\max }=1.05_{-0.22}^{+0.3}\left(m_{\phi} / \lambda^{1 / 4}=6.82_{-0.81}^{+0.87}\right)$, which is roughly in agreement with the findings reported in this work.

Strigari et al. (2008) suggested that dwarf galaxies satellites of the MW, have a common mass within $300 \mathrm{pc}$ of $\sim 10^{7} \mathrm{M}_{\odot}$. They suggest that such a finding could shed light in a characteristic scale for the clustering of dark matter. In Figure 5 the gray shaded region shows Strigari et al. (2008)'s results. Overplotted are the results of this work. The light blue symbols correspond to the $\Lambda=0$ case, and the pink ones correspond to the $\lambda \gg 1$ case. It is encouraging to find that for the best fits of the SFDM model for the M31 and MW dSph galaxies, the mass within $300 \mathrm{pc}$ is in agreement with Strigari et al. (2008) findings.

It has to be noted that the central mass density of the MW's dSphs range from $\rho_{0} \approx 0.03$ to $0.3 \mathrm{M}_{\odot} \mathrm{pc}^{-3}$ (Kormendy \& Freeman 2014; Burkert 2014). It is encouraging that the latter values of the central density are not only in agreement with the central density obtained for the MW's sample $\left(\sim 0.18 \mathrm{M}_{\odot} \mathrm{kpc}^{-3}\right)$, but also with the whole sample $\left(\sim 0.1 \mathrm{M}_{\odot} \mathrm{kpc}^{-3}\right)$.

\section{CONCLUSIONS}

The very high dark-to-stellar mass ratios of dSph galaxies suggest that they are the most DM dominated objects in the universe, and therefore ideal laboratories to test any DM alternative model. In this work I compare the kinematics of $22 \mathrm{dSph}$ galaxies satellites of M31, and 19 dSph galaxies of the MW. I study also a third group of galaxies containing the sum of all M31 and MW dSphs (ALL).

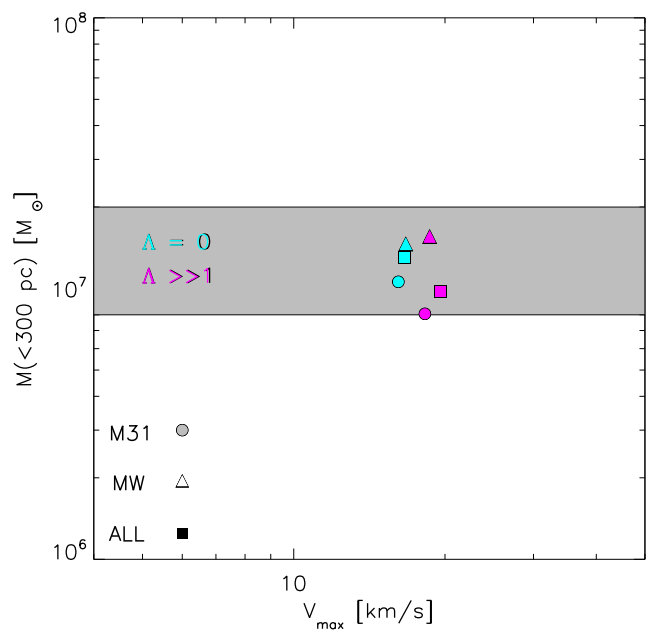

FIG. 5.- The gray shaded region shows the results of Strigari et al. (2008). The resulting mass within 300 pc for M31 (circle), the $M W$ (triangle), and all the sample (square) are shown, for the $\Lambda=0$ case (light blue symbols) and the $\Lambda \gg 1$ case (pink symbols).

I study the hypothesis that all $\mathrm{dSph}$ are embedded in dark matter halos with a same mass Walker et al. 2009; Collins et al. 2014), by fitting the dSph's half-light radius, and the velocity at the half-light radius, to the SFDM model, in two different regimes: when $\Lambda=0$ and when $\Lambda \gg 1$.

There is a very good agreement between the velocity at the half-light radius for the best fit for the ALL sample $(16.4 \mathrm{~km} / \mathrm{s})$ from the $\Lambda=0$ case, with those obtained using an NFW $(16.2 \mathrm{~km} / \mathrm{s})$, and a cored $(15.6 \mathrm{~km} / \mathrm{s})$ DM profile (see Equation 21). This corresponds to a 
mass of the SFDM boson of $\sim 4 \times 10^{-22} \mathrm{eV}$, which is in agreement with our previous findings (Lora et al. 2012; Lora \& Magaña 2014).

A higher value for the velocity at the half-light radius was found for the $\Lambda>>1$ case $(19.6 \mathrm{~km} / \mathrm{s})$ resulting in a $m_{\phi} / \lambda^{1 / 4} \sim 7.6 \mathrm{eV}$.

The M31-dSph galaxies with high luminosities and mass-to-light ratios ranging from 27 to 78 , are better reproduced with the SFDM model than with an NFW or core DM model (see light-blue symbols in Figure 33). Hence, MW-dSph with very high mass-to-light ratios (ranging from 1500 to $\sim 100$ ) and very low values of the luminosity are in better agreement with a NFW/core DM profile than with a SFDM (see dark-blue and purple symbols in Figure 3).

The mass within $300 \mathrm{pc}$ for the SFDM model (for both $\Lambda=0$ and $\gg 1$ ) is in a very good agreement with
Strigari et al. (2008)'s findings. The latter suggests a universal SFDM halo mass for the dSph galaxies in the MW and M31. These results are encouraging, since also a unique mass of the SFDM boson (for the $\Lambda=0$ case) of $\sim 4.8 \times 10^{-22} \mathrm{eV}$ is obtained. One would expect a unique mass of the SFDM boson, and not to have a different SFDM boson mass for different dSph galaxies.

I would like to thank Michelle L. M. Collins and Nicolas F. Martin, for making their data available to me. I also thank Steffen Brinkmann and Colin W. Glass from HLRS, for making their CMA routine available to me. I thank Andreas Just, Juan Magaña and Avon Huxor for very helpful comments and discussions, that gave as a result an improved version of this paper. I gratefully acknowledges support from the Heidelberg University Innovation Fund FRONTIER.

\section{REFERENCES}

Amorisco, N. C. \& Evans, N. W. 2012, MNRAS, 419, 184

Barcelo C., Liberati S. \& Visser M., 2005, Living Rev. Rel., 8, 12

Battaglia, G., Tolstoy, E., Helmi, A., Irwin, M., Parisi, P., Hill, V. \& Jablonka, P., 2011, MNRAS, 411, 1013.

Bernal, A. PhD Thesis.

http://www.fis.cinvestav.mx/thesis/index.php/thesis

Böhmer, C. G. \& Harko, T. 2007, JCAP 06, 25

Boylan-Kolchin, M., Bullock, J. S., \& Kaplinghat, M. 2011b, MNRAS, 415, L40

Boylan-Kolchin, M., Bullock, J. S., \& Kaplinghat, M. 2012, MNRAS, 422, 1203

Burkert, A. 2014, submitted to ApJ, arXiv:1501.06604

Chi-Wai Lai PhD Thesis.

http://laplace.phas.ubc.ca/Members/matt/Doc/Theses/ Phd/lai.pdf

Collins, M. L. M., Chapman, S. C., Rich, R. M., et al. 2013, ApJ, 768,172

Collins, M. L. M., Chapman, S. C., Rich, R. M., et. al, 2014, ApJ, 783,7

Dalfovo F., Giorgini S., Pitaevskii L. P. \& Stringari S., 1999, Rev. Mod. Phys., 71, 463

de Blok W. J. G. \& Bosma, A., 2002, A\&A, 385, 816

Diez-Tejedor, A., Gonzalez-Morales, A. X. \& Profumo, S., 2014, PhysRev, 90, 4, 43517

Guzmán, F.S. \& Matos, T., 2000, CQGr, 17, L9

Guzmán, F. S. \& Ureña-López, L. A., 2004, PRD, 69, 124033

Guzmán, F. S. \& Ureña-López, L. A., ApJ, 2006, 645, 814

Hansen, N. \& Ostermeier, A. 2001, Evolutionary Computation, $9(2), 159$

Hansen, N., Müller, S. D. \& Koumoutsakos, P. 2003, Evolutionary Computation, 11(1):118, 2003

Hu, W., Barkana, R. \& Gruzinov, A.,2000, Phys. Rev. Lett., 85, 1158

Jae-weon Lee \& In-guy Koh, 1996, Phys. Rev., 53, 2236

Jardel, J. R. \& Gebhardt, K., 2012, ApJ, 746, 89

Ji S. U., \& Sin S.-J., 1994, PRD, 50, 3655

Kleyna, J. T., Wilkinson, M. I., Gilmore, G. \& Evans, N. W., 2003, ApJ, 588L, 21

Klypin, A., Kravtsov, A. V., Valenzuela, O. \& Prada, F., 1999, ApJ, 522, 82

Kormendy, J.\& Freeman, K. C. 2014, submitted to ApJ,

arXiv:1411.2170
Lora, V., Sánchez-Salcedo, F. J., A. C. Raga \& A. Esquivel, 2009, ApJ, 699, 113L

Lora, V., Magaña, J., Bernal, A., Sánchez-Salcedo, F. J. \& Grebel, E. K., 2012, JCAP, 2, 11

Lora, V. \& Magaña, J., 2014, JCAP, 9, 11

Martin, N. et al. In Prep. 2015

Matos, T., Guzmán F. S. \& Ureña-Lopez, L. A., 2000, Class. Quant. Grav., 17, 1707

Matos, T., Vazquez-Gonzalez, A. \& Magaña, J. 2009, MNRAS, 393, 1359

Magaña, J. \& Matos, T., 2012, JPhCS,378, 012012

Magaña, J., Matos, T., Suarez, A. \& Sanchez-Salcedo, F. J. 2012, JCAP, JCAP10(2012)003

McConnachie, A. W. 2012, AJ, 144, 4

Navarro J. F., Frenk C. S. \& White S. D. M., 1996, ApJ, 462, 563

Peebles, P.J.E., 1999, PRD, 60, 103506

Pitaevskii, L. P. 1961, Sov. Phys., JETP 13, 451

Read, J. I., Wilkinson, M. I., Evans, N. W., Gilmore, G., \& Kleyna, J. T., 2006, MNRAS, 367, 387

Robles, V. H. \& Matos, T., 2012, MNRAS, 422, 282

Rodríguez-Montoya, I., Magaña, J., Matos, T. \& Pérez-Lorenzana, A., 2010, ApJ, 721, 1509

Ruffini, R. \& Bonazzola, S., 1969, PR, 187, 1767

H. -Y. Schive, T. Chiueh \& T. Broadhurst, 2014, Nature, 10, 496

Seidel, E. \& Suen, W.-M., 1991, PRL, 66, 1659

Sin S. J., 1994, PRD, 50, 3650

Strigari, L. E., Bullock, J. S., Kaplinghat, M., Simon, J. D., Geha, M., Willman, B. \& Walker, M. G. 2008, Nature, 454, 1096

Walker, M. G., Mateo, M., Olszewski, E. W., Pal, J. K., Sen, B. \& Woodroofe, M., 2006, ApJ 642, L41

Walker, M. G., Mateo, M., Olszewski, E. W., et al. 2009, ApJ, 704,1274

Walker, M. G. \& Peñarrubia, J. 2011, ApJ, 742, 20

Wang X. Z., 2001, Phys. Rev. D 64, 124009

Wolf, J., Martinez, G. D., Bullock, J. S., et al. 2010, MNRAS, 406, 1220

van den Bosch, F. C., Robertson, B. E., Dalcanton, J. J. \& de

Blok, W. J. G., 2000, AJ, 119, 1579

\section{APPENDIX}

\section{THE EVOLUTION STRATEGY WITH COVARIANCE MATRIX ADAPTATION OPTIMIZATION}

The Evolution Strategy with Covariance Matrix Adaptation (CMA-ES) is a stochastic, derivative-free method for numerical optimization (Hansen \& Ostermeier 2001; Hansen et al. 2003). It is robust and superior to derivative based search methods in rugged search landscapes, i.e. containing noise, outliers, large derivatives etc. 


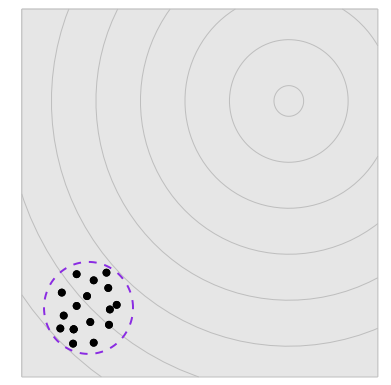

First Generation

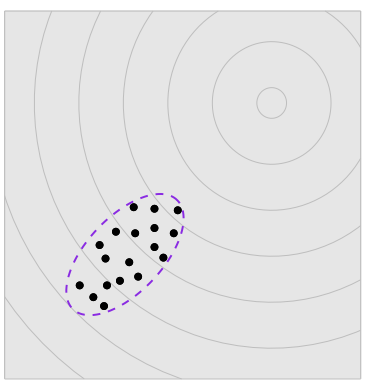

Second Generation

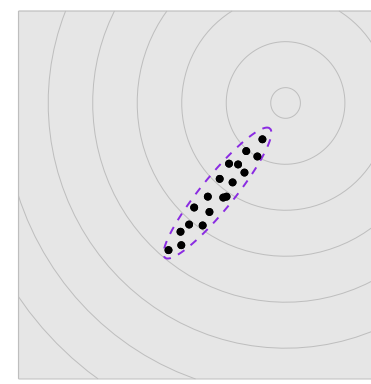

Third Generation

FIG. 6.- Representation of the CMA optimization method.

An evolutionary strategy algorithm uses mutation and selection on a given sample of solutions until a termination criterium is met. More precisely, the algorithm is initialised with a population of $\mu$ candidate solutions in the solution space, called parents. From these parents, $\lambda \geq \mu$ new candidate solutions (children) are created by mutation, i.e. by scattering their properties (=genes) around the parents properties:

$$
\mathbf{x}_{i} \sim \sigma \mathcal{N}_{i}(\mathbf{m}, \mathcal{C}) \text { for } i=1, \ldots, \lambda .
$$

In the latter Equation $\mathbf{x}_{i}$ is the property vector of child $i, \mathbf{m}$ is the weighted mean property vector of the parents, $\sigma$ is the step size, $\mathcal{N}$ is the normal distribution around $\mathbf{m}$, which is distorted by the covariance matrix $\mathcal{C}$. The children are evaluated by a fitness function $\left(f(\mathbf{x}): \mathbb{R}^{n} \rightarrow \mathbb{R}\right)$ and only the fittest $\mu$ children become the parents of the next evolutionary step.

CMA-ES enhances the evolutionary approach by updating the step size and the covariance matrix independently after each evolutionary step. For that purpose, the algorithm takes into account the $\mu$ fittest children according to a given fitness function, the distance and direction of the new weighted mean relative to the old one, and lastly, how many children achieved a better fitness than the best parent.

The mean of the distribution is updated such that the likelihood of previously successful candidate solutions is maximized. The covariance matrix of the distribution is incrementally updated such that the likelihood of previously successful search steps (mutations) is increased. Both updates can be interpreted as a natural gradient descent. See Figure 6 .

The adaptive algorithm design makes CMA-ES a black-box optimization strategy in the sense that no information about the solution landscape is required. The algorithm is furthermore robust against transformations of the solution space, e.g. by exchanging the order of parameters in the fitness function or different dimensional scaling of the solution space. Dependences between the solution candidate properties influence the covariance matrix, thus accelerating the search for the optimal solution. 